\title{
Promoting Remyelination in Multiple Sclerosis—Recent Advances
}

\author{
E. Jolanda Münzel · Anna Williams
}

Published online: 16 November 2013

(C) The Author(s) 2013. This article is published with open access at Springerlink.com

\begin{abstract}
We review the current state of knowledge of remyelination in multiple sclerosis (MS), concentrating on advances in the understanding of the pathology and the regenerative response, and we summarise progress on the development of new therapies to enhance remyelination aimed at reducing progressive accumulation of disability in MS. We discuss key target pathways identified in experimental models, as although most identified targets have not yet progressed to the stage of being tested in human clinical trials, they may provide treatment strategies for demyelinating diseases in the future. Finally, we discuss some of the problems associated with testing this class of drugs, where they might fit into the therapeutic arsenal and the gaps in our knowledge.
\end{abstract}

\section{Introduction}

Multiple sclerosis (MS) is the most common cause of nontraumatic disability in young people, affecting approximately 2.5 million people worldwide [1]. Despite much research effort, the aetiology is not fully understood, and may include genetic susceptibility, epigenetic regulation and environmental factors [2]. MS is a disease of the central nervous system (CNS), and therefore neurological impairment can include visual, motor, sensory, coordination or cognitive deficits depending on the site of damage $[3,4]$. Most patients initially present with relapsingremitting disease (RRMS), where periods of symptomatic

E. Jolanda Münzel · A. Williams ( $₫)$

MRC Centre for Regenerative Medicine,

University of Edinburgh, Edinburgh Bioquarter,

5 Little France Drive, Edinburgh EH16 4UU, UK

e-mail: annacwilliams@yahoo.co.uk; anna.williams@ed.ac.uk impairment are followed by recovery. This phase can last for years; however, eventually most patients will progress to a secondary progressive (SPMS) disease course, where residual disability gradually accrues over time. Some patients steadily accumulate disability with no recovery from the very onset of MS; this is known as primary progressive MS (PPMS).

These different phases of the disease reflect the underlying pathology, which is at first more inflammatory and demyelinating (in the relapsing and remitting phase) and then more neurodegenerative (in the progressive phase). Currently licensed disease-modifying drugs for MS are anti-inflammatory; they suppress the immune system and reduce relapse rates but are ineffective in progressive disease. There are as yet no progressive disease-modifying drugs to slow, stop or reverse neurodegeneration. Neuroprotective drugs have been sought for many other neurological diseases, including stroke, spinal cord injury and Alzheimer's disease, with limited success. However, an alternative strategy is available in MS, where neuroprotection may be enhanced through myelin repair-remyelination. In this review, we outline the recent advances made in our understanding of how myelin is lost in MS, what prevents remyelination in humans from being efficient, and how we might be able to manipulate remyelination to enhance it to treat progressive MS.

\section{Pathology of Multiple Sclerosis}

The pathological hallmarks of all MS subtypes are focal areas or 'plaques' of demyelination in the CNS, with surrounding inflammation and neurodegeneration. Two hypotheses aim to explain the underlying pathology of MS: first, that inflammation induces demyelination, which leads 
to secondary axon degeneration; or second, that MS is a neurodegenerative disease with secondary inflammation and demyelination.

Classically, MS has been thought of as an autoimmune disease. Self-antigens (as yet mostly unknown) presented by major histocompatibility complex class II antigen-presenting cells (e.g. dendritic cells and macrophages) are misidentified as being foreign and there is activation of auto-reactive $\mathrm{T}$ cells $[5,6]$. These auto-reactive $\mathrm{T}$ cells cross the blood-brain barrier, and cause inflammatory damage through expression of inflammatory cytokines and reactive oxygen species and attraction of other immune cells like macrophages and B cells, creating a loop of proinflammatory enhancement, which causes demyelination and axonal degeneration. In MS, transient gadoliniumenhancing brain lesions are seen on magnetic resonance imaging (MRI), confirming blood-brain barrier breakdown, and oligoclonal immunoglobulin $\mathrm{G}$ (IgG) bands are seen in the cerebrospinal fluid (CSF) (but not the serum), suggestive of a B-cell response to antigens within the CNS [7]. Demyelination of axons removes saltatory conduction, physical protection and metabolic support [8, 9] of the axon and causes neurodegeneration. Supporting this, more axonal damage is seen in lesions with a high degree of acute inflammation $[10,11]$ and is also a consequence of chronic demyelination [12, 13]. However, histopathological analysis of post mortem brains from MS patients also shows that axonal damage occurs not just in active demyelinating lesions but can also be present in lesions that show signs of remyelination [14]. In addition, axonal injury is already extensive in early stages of the disease and decreases with time [15], and this is supported by the coexistence of inflammatory and neurodegenerative biomarkers early in MS [16].

Therefore, is MS instead primarily a neurodegenerative disease? In MS, there are widespread grey matter lesions showing signs of neurodegeneration but less immune cell infiltration and demyelination than in white matter lesions [17], and the load of cortical grey matter lesions correlates with changes suggesting diffuse axonal loss in normalappearing white matter of MS patients detected on MR sequences [18]. Neurodegeneration occurs early and in widespread areas of the brain distant from white matter lesions [19]. The extent of neurodegeneration also does not always correlate with the amount of demyelination-for example, there is more axonal loss in spinal cord plaques from patients with PPMS than in those from patients with SPMS, despite more extensive demyelination in SPMS patients [20]. Furthermore, progressive disability correlates more with neurodegeneration (and brain atrophy) than white matter demyelination [21, 22].

Whatever the order of pathological events, it is clear that preventing inflammation, demyelination and neurodegeneration, or enhancing remyelination and neuroprotection, are all potential targets for therapies to reduce disease and disability in MS. Licensed drugs that are effective at reducing inflammation and demyelination in MS are now available, but no therapies exist to reduce, stop or reverse neurodegeneration. Regardless of whether axonal loss is the primary event or occurs secondary in the disease pathology of MS, it is a major contributor to accumulation of permanent disability [23] and it is untreatable. There are two ways to approach neuroprotective therapy in MSeither to use agents to directly protect axons, or to improve remyelination, which restores the support of axons.

\section{How Does Remyelination Mediate Neuroprotection?}

Myelin is formed by oligodendrocytes in the CNS by extension and wrapping of their plasma membrane processes around neuronal axons in a spiral fashion. Each axon has multiple short segments of myelin (internodes) that are separated by gaps (nodes of Ranvier), where sodium channels are concentrated and which facilitate fast saltatory nerve conduction. As well as providing an electrical insulating sheath, oligodendrocytes provide the axons with metabolic support via channels within the myelin [8, 9]. Therefore, demyelination leads to both decreased nerve conduction velocity, resulting in neurological impairment, and predisposition of the axons to neurodegeneration of the axons to neurodegeneration due to lack of physical and metabolic support. Demyelinated axons may also have a higher metabolic demand, as electrogenic machinery along the axons, such as sodium channels, no longer cluster at the nodes of Ranvier, and nerve impulse conduction becomes less efficient [24, 25]. If this increased metabolic demand is not met by mitochondria, which also have to reorganise in a demyelinated axon, the production of nitric oxide is thought to further compromise axonal integrity [26, 27]. This hypothesis is supported by the fact that in MS brain, axonal degeneration is most prevalent in actively demyelinating lesions where inflammation is greatest, and that sodium channels in these areas are found diffusely located along demyelinated axons [28].

Remyelination may reverse or prevent some of these structural and metabolic changes observed in axons after demyelination. Studies in rodent models of MS using toxin-mediated demyelination showed that remyelinating cells were able to restore the normal pattern of electrogenic machinery, in particular, clustering of sodium channels in nodes of Ranvier and potassium channels in the juxtaparanode [29]. Furthermore, increases in mitochondrial content and clustering of nodal proteins were found in remyelinated but not demyelinated axons in human and rodent tissues, suggesting that remyelination can also 
restore metabolic changes that occur when axons are demyelinated [30, 31]. Neuroprotection through remyelination has been explored in a mouse model of experimental demyelination, in which oligodendrocyte precursor cells with the capacity to remyelinate were killed by irradiation and this resulted in reduced myelin repair and increased axonal loss. However, transplantation of neural progenitor cells into the irradiated and demyelinated area rescued both remyelination and axonal survival [32]. Moreover, CNS remyelination in various experimental animal models is associated with functional recovery [33-35]. Studies in humans to support these findings in rodents are difficult, as remyelination is difficult to measure at high resolution in vivo, but there is supportive evidence from post mortem MS patient brain tissue studies that shows that remyelination can be extensive in subsets of MS patients [36], and remyelinated lesions exhibit less acute axonal injury than active demyelinating lesions [15].

However, perhaps remyelination is not sufficient to fully prevent axonal loss. We know that internodal lengths are shorter in remyelinated axons (resulting in more sodium channel clusters per length of nerve) and that mitochondrial changes are not fully reversed [31]. There is also no doubt that axonal degeneration can occur without areas of demyelination (for example, see Bitsch et al. [14]), and this could be either through a separate mechanism or perhaps through related anterograde and retrograde degeneration of axons damaged in a demyelinated plaque. Direct inflammatory damage by activated macrophages and microglia may also occur, as these have also been found outside of active lesions in the normal-appearing white matter in the CNS [37]. The question of whether remyelination can protect axons long term was asked in a mouse model of demyelination using the toxin cuprizone, where there was full remyelination and functional recovery initially, but a late functional decline, concurrent with axonal loss in affected white matter tracts and changes in myelin composition [38]. However, cuprizone also damages axons [39], which may well have contributed to these changes.

Therefore, the debate still exists on whether remyelination completely restores normal axonal health and prevents neurodegeneration. However, it does appear to restore much function and at least limit degeneration, and so enhancing this process has become a key therapeutic aim in MS, to try and avoid or treat progressive disease.

\section{The Steps Necessary for Remyelination}

To develop a therapy to enhance remyelination requires knowledge of the biology involved. Myelinating oligodendrocytes during development originate from oligodendrocyte precursor cells (OPCs). OPCs are still present in the brain parenchyma and in progenitor zones in the mature adult CNS and are the source of oligodendrocytes that mediate remyelination of demyelinated axons [40], coming either from within the lesion or from the immediately surrounding white matter [41], or from the subventricular zone (the site of neurogenesis in the adult brain), if the lesion site is in close proximity [42, 43]. This population of progenitor cells is likely responsible for generating almost all remyelinating cells in demyelinating lesions in the adult CNS according to fate mapping of OPCs in mice [44]. Preexisting mature oligodendrocytes are unlikely to contribute to remyelination, either directly or by de- and re-differentiating (similarly to Schwann cells in the peripheral nervous system [PNS] [45]), as previous studies have reported a lack of remyelination capacity of post-mitotic oligodendrocytes, even if they survive within a demyelinated lesion or are transplanted from a normally myelinating area into a lesion site [46, 47]. Conversely, rat OPCs transplanted into demyelinated areas can differentiate into myelinating oligodendrocytes and produce robust myelin [48]. Furthermore, dividing endogenous progenitor cells in the adult rat brain have been shown to respond to demyelination by differentiating into myelin-forming oligodendrocytes [49].

In early stages of MS, spontaneous remyelination does occur, and it can be reasonably extensive in some patients $[36,50]$, but this declines with age [51]. For successful remyelination to take place, OPCs need to be able to respond to demyelination by proliferation, migration to the lesion site, differentiation and ensheathment of axons (as described in Fig. 1). Deficient remyelination can therefore occur through a failure at any of these steps. From study of post mortem MS brain, it has been estimated that around $70 \%$ of MS lesions that remain demyelinated contain plentiful OPCs, suggesting a failure of differentiation and the other $30 \%$ contain few or no OPCs, indicating failure of recruitment $[3,52]$. Therefore, manipulations to improve either or both of these processes may be beneficial for remyelination. The process of remyelination does not solely involve oligodendroglial cells, and, increasingly, other cells in the vicinity are being implicated. Microglia/ macrophages are needed to clear myelin debris following demyelination, which is known to be inhibitory for remyelination, at least in rodents [53], and secrete substances to create a favourable environment for precursor cell maturation and remyelination [54]. Astrocytes have also been implicated in both providing useful factors for remyelination and impeding it, with astrocytic scarring [55].

To test ways of manipulating and improving remyelination, we use animal models, mostly in rodents. However, most animal models of demyelination show a robust and complete remyelination response, and so to this extent they poorly model humans, where remyelination capacity is limited. Although myelin is replaced around virtually all 


\section{Lesion}
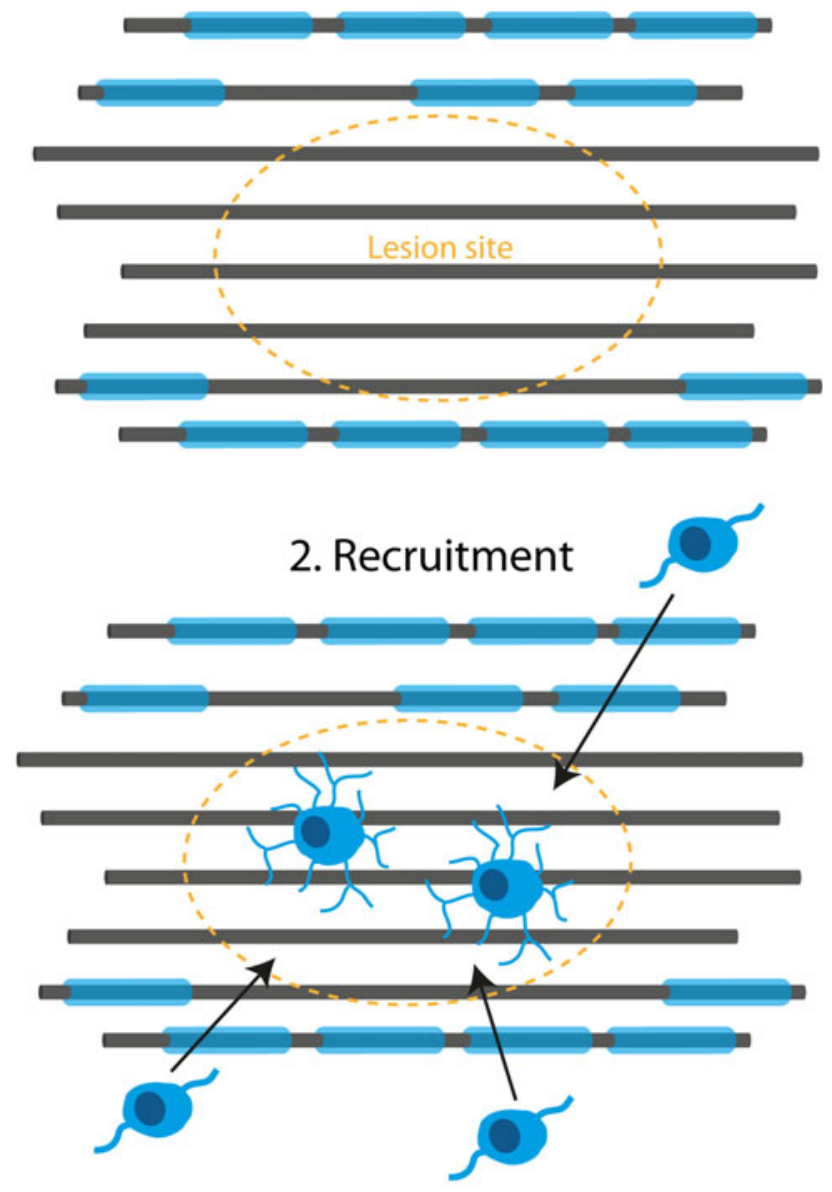

\section{Differentiation}

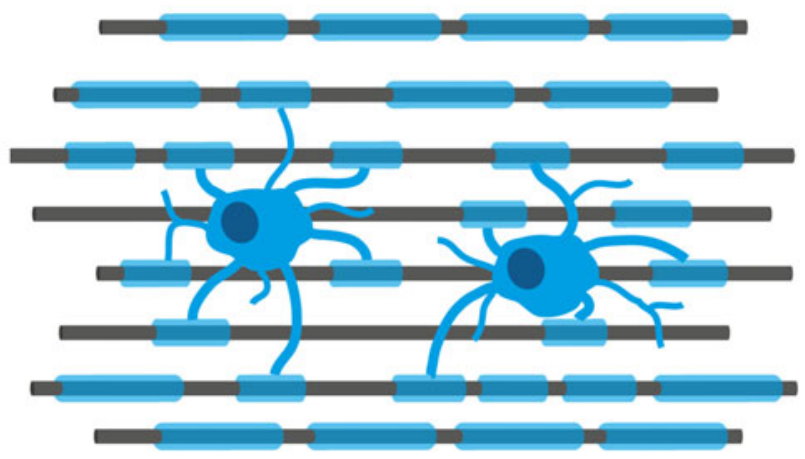

Fig. 1 Major steps required for remyelination. For successful remyelination, oligodendrocyte precursor cells need to be recruited to the lesion site and then differentiate into myelinating oligodendrocytes. This is a very intricate process that can fail if not regulated effectively

axons after demyelination in rodents, remyelinated myelin sheaths are not fully restored to normal, as they are thinner and with shorter internodal lengths compared with developmental myelin sheaths in both rodents and humans
[56-60]. In spite of this, remyelinated axons do conduct electricity using saltatory conduction, albeit at a somewhat slower velocity, but this seems sufficient to restore function in animal models [33-35].

\section{Promoting Remyelination-Potential Candidate Drugs}

How can we intervene to improve remyelination and maintain function in MS patients? All current treatments used to treat MS in clinical practice act primarily on modulating or suppressing the immune system to prevent further relapses, but they do not delay or slow disease progression or affect remyelination. The current licensed treatments (in the UK) are the beta-interferons, glatiramer acetate, natalizumab and fingolimod. Natalizumab (a monoclonal antibody blocking $\alpha 4$ integrin and preventing $\mathrm{T}$ cells from crossing the blood-brain barrier) and fingolimod (a sphingosine-1-phosphate receptor modulator preventing $T$ cells from exiting lymph nodes) are both also in clinical trial to test whether they prevent or slow progression of disease (NCT01416181, NCT00731692), but they are not being directly tested for their ability to promote remyelination. Fingolimod does promote rodent oligodendrocyte process extension and survival in vitro [61, 62]; however, it remains unclear how it affects regeneration of myelin, as although increased remyelination has been demonstrated in the multi-cellular environment of ex vivo rodent slice culture preparations [63], it was not seen in in vivo rodent studies [64]. While one human clinical trial has reported no effect of fingolimod on disease progression in RRMS patients [65], other trials continue (NCT00731692, NCT01498887). The wealth of other drugs designed to reduce MS relapses (including daclizumab, teriflunomide, ocrelizumab, laquinimod and siponimod), which are in clinical trial, awaiting licensing, or that have received licensing in some countries, have also not been shown to affect disease progression or remyelination. However, alemtuzumab (now approved by the European Medicines Agency) and dimethyl fumarate/ BG-12 (approved in Europe and the USA) do appear to have an effect on reducing disease progression as outlined below. Furthermore, clinical trials have started with anti-LINGO-1 antibodies and the human monoclonal IgM antibody number 22, which have been designed to enhance remyelination.

\subsection{Alemtuzumab}

Alemtuzumab is a monoclonal antibody that destroys lymphocytes via CD-52 recognition and is currently used in the treatment of T-cell lymphoma and chronic lymphatic leukaemia [66, 67]. Two randomised controlled phase III clinical trials investigating the efficacy of alemtuzumab 
compared with interferon-beta 1a in patients who had not received other primary treatment (CARE-MS I [Comparison of Alemtuzumab and Rebif Efficacy in Multiple Sclerosis]) and who had failed on other disease-modifying medication (CARE-MS II) were recently completed. Both trials showed that patients on alemtuzumab treatment were less likely to experience a relapse over the course of the 2 years [68, 69]. In addition, patients treated with alemtuzumab in CARE-MS II (only) showed a $42 \%$ reduction in sustained accumulation of disability. The mechanism for this reduction in disability progression may be directly related to fewer damaging episodes of demyelination, or indirectly allowing more time for remyelination to occur spontaneously, or directly by an effect on neuroprotection/remyelination. One possible support for the latter is that cultures of peripheral blood mononuclear cell cultures, in particular $\mathrm{T}$ cells, produce increased concentrations of potentially useful neuronal growth factors when treated with alemtuzumab and stimulated with myelin basic protein. In addition, media from these cell cultures promote survival of neurons and OPCs and enhance oligodendrocyte differentiation and myelination [70]. Future clinical trials are planned using MRI analysis to measure myelin repair for further exploration of the putative neuroprotective and remyelinating potential of alemtuzumab (NCT01307332, NCT01395316).

\subsection{Dimethyl Fumarate}

Dimethyl fumarate is the methyl ester of fumaric acid and has recently been approved by the US Food and Drug Administration (FDA) and European Medicines Agency for treatment of MS. It is both immunomodulatory and upregulates the transcription factor Nrf2 [71], but its precise mode of function is unknown. Newly completed phase III clinical studies have indicated its ability to significantly reduce relapse rate and development of brain lesions in patients with RRMS [72, 73]. In addition, these trials showed a reduced risk of disability progression at 2 years and a favourable safety and tolerability profile. However, while there is no direct evidence that dimethyl fumarate has effects on remyelination and neuroprotection in either humans or experimental models of MS, the related antipsychotic compound quetiapine, a fumarate salt, does possess pro-remyelination and neuroprotective properties in rodents [74]. There is an increase in differentiation of rodent progenitor cells into oligodendrocytes and a greater extent of myelination in cortical aggregate cultures after treatment with quetiapine [75]. Moreover, findings from in vivo experimental models demonstrate reduced demyelination and loss of oligodendrocytes [76, 77] with quetiapine treatment, as well as faster return of myelin proteins [78]. These data suggest that quetiapine enhances remyelination in experimental models, raising hopeful possibilities for the related compound dimethyl fumarate in humans.

\subsection{Anti-LINGO-1 Antibodies}

LINGO-1 is a protein containing N-terminal leucine-rich repeat domains that interacts with the Nogo receptor and has been shown to regulate axon outgrowth [79]. Recent research revealed that LINGO-1 plays a key role in negatively regulating OPC differentiation and remyelination in rodent models of MS; therefore, blocking this may be effective in promoting remyelination in the clinical setting. LINGO-1 gene knockout in mice, or treatment with an anti-LINGO-1 antagonist antibody, resulted in enhanced functional recovery from inflammatory autoimmune demyelination and also increased remyelination [80]. Similarly, the anti-LINGO-1 antagonist also showed increased remyelination in toxin-induced demyelinating lesions in rats, by direct stimulation of OPC differentiation [81, 82]. A recent preclinical study in an immune model of demyelination in rats (MOG-EAE), thus far only published in abstract form, also demonstrates a fivefold reduction of axonal loss in the optic nerve of rats treated with BIIB033 (an anti-LINGO-1 antibody) compared with control antibody, which became more marked (eightfold reduction) in combination with methylprednisolone [83]. A clinical phase Ib study compared the effect of BIIB033 (an antiLINGO-1 antibody) in patients with RRMS in a placebocontrolled randomised trial (NCT01244139); this is the first trial targeted at directly improving remyelination in MS. The study was completed in April 2012, showing no serious adverse events, as reported at the American Academy of Neurology annual meeting. A phase II trial is planned and will give additional information with regards to safety, tolerability and efficacy (NCT01864148).

\subsection{Human Monoclonal IgM Antibody 22}

The human monoclonal IgM antibody 22 (rHIgM22) was initially discovered in the Mayo Clinic (Rochester, MN, USA), where researchers found that this recombinant antibody, usually present in the serum of human MS patients, was able to induce spinal cord remyelination in a virus-mediated mouse model of demyelinating disease [84]. Compared with control-treated animals, mice that received rHIgM22 exhibited fewer lesions $(34.3 \%$ [rHIgM22] vs. $41.8 \%$ [control]) and increased remyelination (59.7\% [rHIgM22] vs. $15.8 \%$ [control]). Similar increases in remyelination were reported in MRI studies using the same experimental animal model [85]. Further research suggests that this pro-remyelination effect of $\mathrm{rHIgM} 22$ is independent of immunomodulation [86] and is associated 


\section{Promoting factors \\ Semaphorin $3 \mathrm{~F}$ \\ CXCL 1, 8, 10 \\ PDGF}

Mechanism

Inhibiting factors

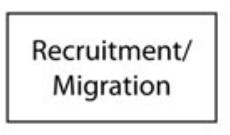

Semaphorin $3 \mathrm{~A}$ Migration

\section{Retinoic acid \\ CXCL 12}

Endothelin 2

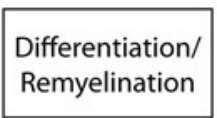

Lingo-1

Notch1

Wnt/ß-catenin

Hyaluronan

BMP

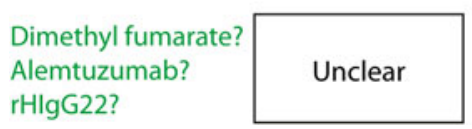

Fig. 2 Selected factors that influence remyelination and their mechanism of action. Several factors/signalling pathways, which positively (green) or negatively (red) influence remyelination by altering known necessary steps during the remyelination process have already been identified. $B M P$ bone morphogenic proteins, $P D G F$ platelet-derived growth factor

with anti-apoptotic signalling in pre-myelinating oligodendrocytes [87]. Recent data provide evidence of potential mechanisms underpinning the remyelination-enhancing effect of rHIgM22, involving the Src family kinase Lyn and platelet-derived growth factor (PDGF)-receptor alpha signalling [88, 89]. A phase I clinical trial is now recruiting patients to evaluate safety, tolerability, pharmacokinetics and immunogenicity of a single intravenous dose of rHIgM22 in patients with MS (NCT01803867). This study may provide a first indication of whether $\mathrm{rHIgM} 22$ has similar pro-myelinating effects in human MS patients. The estimated end date of the trial is September 2014.

These four compounds, two of which are primarily antiinflammatory but may also aid remyelination, and two of which have been designed to enhance remyelination, are likely to be the first of many (Fig. 2). LINGO-1 was one of the first molecules shown to aid remyelination in the labarotory, in 2007, and is now in clinical trial. Other molecules that have since been discovered are making their way in the same direction, and either promote endogenous remyelination by improving OPC migration or OPC differentiation or involve cell transplantation. We discuss a selected number of interesting candidates below.

\section{Pre-Clinical Approaches to Enhancing Remyelination}

\subsection{Enhancing Endogenous Remyelination}

To enhance endogenous remyelination, research has therefore focussed on the two essential steps-to repopulate the demyelinated area with OPCs and to promote their remyelinating ability — and several factors/pathways have already been identified (Fig. 2).

\subsubsection{Aiding Migration of Oligodendrocytes}

At least $30 \%$ of MS lesions lack OPCs, pointing towards insufficient OPC recruitment $[3,90]$. One research goal has therefore been to identify targets that will aid the migration of OPCs to the demyelinated area. PDGF $\alpha$ is the archetypal chemoattractant for OPCs, although it is difficult to separate this effect from its effect on OPC proliferation. OPCs express the PDGF $\alpha$ receptor, and repopulate the lesion site during the early remyelination process in successful remyelination in a mouse model [91]. In a rat model with toxin-induced demyelination, addition of PDGF increased both OPC number (mitogenic) and presence in lesions though with no increases in remyelination [92]. However, using the same model but with a different delivery system of PDGF resulted in an increased number of oligodendrocytes and enhanced remyelination [93]. Thus, improving the number of OPCs in the correct place may help, but in animal models where remyelination is normally robust and does not appear to fail due to insufficient OPC recruitment, this may be difficult to ascertain. A link may exist between PDGF and the human monoclonal IgM (rHIgM22), which is currently in clinical trial as described above. Evidence from in vitro studies suggests that activation of the PDGFalpha receptor by rHIgM22 is necessary for stimulation of OPC proliferation and promotion of remyelination [89].

The semaphorins have also been identified as potential targets to promote OPC recruitment. These are a class of secreted and transmembrane proteins that were primarily described as axonal guidance molecules in the developing nervous system. Class 3 semaphorins have also been identified as chemotactic factors for oligodendroglial cells during development. Semaphorin 3A (Sema3A) and 3F (Sema3F) aid the recruitment of glial precursor cells from the brain to the developing optic nerve by their repulsive and attractive properties, respectively [94, 95]. These molecules are down-regulated in normal brain white matter but are re-expressed after injury, including demyelination in rodents and humans [96]. Moreover, expression of the semaphorin 3 receptors, neuropilins and plexins, is increased on OPCs after demyelination in mice, and upon Sema3A/3F overexpression around lesions, OPC recruitment to lesions was reduced or increased, respectively [97]. In human MS post mortem tissue, those lesions expressing the chemorepellent Sema3A were more likely to contain few OPCs and not to show remyelination [52]. Furthermore, in a model of focal toxin-induced demyelination in the mouse corpus callosum, addition of recombinant Sema3A to the lesion decreased OPC recruitment and remyelination, and $\mathrm{rSema} 3 \mathrm{~F}$ addition or use of a Sema3A 
knockdown mouse increased OPC recruitment and remyelination. These results give us two new therapeutic targets for promotion of remyelination.

Other chemokines that are able to influence immune cell migration have also been detected around demyelinating lesions. Omari et al. [98, 99] found that immature and more mature OPCs expressed chemokine receptors CXCR1-3 in vitro. The respective ligands CXCL8, 1 and 10 were absent from normal CNS tissue, but were detected in reactive astrocytes bordering MS lesions, providing possible cues for OPC recruitment. Overexpression of CXCL1 led to a milder disease course, decreased neurodegeneration and more prominent remyelination in an immunemediated mouse model of MS [100], supporting a potential pro-migratory, pro-myelinating and neuroprotective function of CXCL1.

Thus, a new group of pro-migratory or anti-repellent molecules have been generated to be investigated further for therapies.

\subsubsection{Promoting Oligodendrocyte Differentiation}

Around $70 \%$ of demyelinated MS lesions contain immature oligodendroglial cells that appear to be in an arrested state, unable to fully differentiate $[3,90]$. As well as the LINGO-1 molecule, discussed above, there are now more than ten targets in this group. These have been identified either due to their importance in developmental myelination or from expression screens of demyelinated tissue. Two pathways known to be important in developmental myelination are the Notch-1 and Wnt pathways. The Notch-1 receptor ligand Jagged-1 is expressed by reactive astrocytes around injury sites and was first identified as a potential negative regulator of remyelination in a microarray screen [101]. During development, Notch-Jagged signalling inhibits OPC differentiation; with Notch-1-null oligodendroglial cells exhibiting accelerated oligodendrocyte differentiation and myelination [102, 103]. Notch-1 also appears to be a key regulator of remyelination as Notch-1 and its effector Hes5 are detected in OPCs in demyelinating lesions in rodent models in vivo, with Notch signalling hindering OPC maturation and myelin formation in vitro [104]. Moreover, transforming growth factor (TGF)-B, a cytokine upregulated in MS, was able to trigger Jagged-1 expression in reactive human astrocytes, which are known to border MS plaques lacking remyelination, while Notch-1 and the downstream effector gene Hes5 localised to immature oligodendroglial cells [101, 105].

Similarly, the Wnt pathway negatively regulates developmental myelination. In transgenic mice overexpressing ß-catenin, a protein complex acting as an intracellular signal transducer in the Wnt pathway, there is no impairment of embryonic development of oligodendrocytes but later differentiation is affected, resulting in fewer oligodendrocytes expressing mature myelin markers (PLP) in the white matter [106]. Consequently, in these mice, electron microscopic analysis at postnatal day 15 (P15) showed hypomyelination of axons, which reached normal wild-type levels by P50, suggesting that Wnt signalling delays rather than blocks oligodendrocyte maturation. Similarly, experimental toxin-induced demyelination in these transgenic mice also resulted in delayed oligodendrocyte differentiation during remyelination [118]. Tcf4, an intranuclear binding partner of B-catenin that is normally detected in developing mice but not in adult white matter, is re-expressed upon white matter demyelination in adult animals [106, 107]. As Tcf4 locates to OPCs, which are recruited to the lesion site in experimental models, and as it is also highly expressed in MS lesions, Tcf4 may also play a role in regulating oligodendrocyte differentiation and myelin repair through active Wnt signalling.

A further molecule critical in development is sonic hedgehog (Shh), which is essential for oligodendrocyte specification. Studies in the adult brain have shown that Shh is still expressed in certain areas, for maintenance of stem cell niches, which show reduced cell proliferation in vivo if Shh signalling is blocked [108, 109]. Viral application of Shh into the lateral ventricle of adult mice results in increased OPC proliferation, with subsequent differentiation, suggesting that overexpression of Shh modifies the stem cell niche to increase the production of precursor cells, which will ultimately differentiate along the oligodendroglial lineage [110]. In experimental demyelinating lesions, Shh upregulation in oligodendroglial cells results in increased OPC proliferation and a Shh antagonist impairs myelin repair [111].

To discover new pathways involved, expression screens have been used. Microarray analysis of demyelinated lesions from rat brain at different stages of remyelination identified the nuclear retinoid $\mathrm{X}$ receptor $(\mathrm{RXR})-\gamma$ pathway as a positive regulator of endogenous remyelination [112]. OPCs express this receptor, and treatment of OPCs with an RXR $-\gamma$ antagonist in vitro leads to impaired OPC maturation, whereas incubation with 9-cis-retinoic acid, a RXR agonist, stimulates differentiation, myelination and remyelination in culture. Furthermore, focal demyelinating lesions in RXR- $\gamma$ knockout mice show accumulation of immature oligodendrocyte lineage cells, and treatment of rats with the RXR- $\gamma$ agonist improves remyelination [112]. Small molecule agonists of this receptor are currently being sought.

Another screen to identify molecules increased in inflammation that aid myelination has identified endothelin 2 as a stimulus for remyelination [113]. Endothelin 2 enhances OPC differentiation, and promotes myelination and remyelination in ex vivo explant cultures. The 
endothelin receptor type $\mathrm{B}$ is expressed by rodent and human oligodendroglial cells [114, 115] and by human oligodendroglial cells in MS brain lesions. Treatment of rats with the agonist of this receptor after a focal demyelinating lesion led to increased remyelination by enhancing differentiation of oligodendrocytes [119].

Other molecules involved include the chemokine CXCL12 and its receptor CXCR4, which promote migration, proliferation and differentiation of neural precursors and, if inhibited, reduce remyelination [116]. The bone morphogenic proteins (BMPs) 4, 6 and 7 are up-regulated in immune-mediated demyelinating lesions in mice [117], and inhibition of BMP4 increases remyelination in areas of focal CNS demyelination in mice [118]. However, this success may also be related to BMP-mediated astrogliosis. The extracellular matrix protein hyaluronan is expressed in human MS lesions and, in immune-mediated demyelinated lesions in mice, hyaluronan blocks OPC differentiation in vitro and in vivo, and may be mediated through the Tolllike receptor (TLR)-2, as this receptor is strongly expressed by oligodendrocytes and TLR2-agonists are able to inhibit OPC differentiation in vitro [119]. Moreover, neutralising antibodies of TLR2 have been shown to prevent hyaluronan-mediated failure of OPC differentiation and TLR-null mice exhibit faster and more effective remyelination.

It is clear even from this selected list that remyelination is not regulated by one single molecule but instead through a combination of signalling pathways acting on OPCs and oligodendrocytes, but also no doubt on the other cell players, the microglia/macrophage, astrocytes and even the blood vessels. There is much to understand about this pathology and regenerative response, but at least the current targets have been identified, and can now be evaluated in further studies moving towards human clinical trials.

\subsection{Transplantation}

An alternative to promoting endogenous remyelination is to transplant exogenous OPCs into the CNS to remyelinate. This approach has been investigated in spinal cord injuryinduced demyelination, and transplantation of OPCs following contusion or irradiation of the rat spinal cord leads to increased remyelination and functional recovery [120, 121]. Similarly, recent studies show that human neural stem cell transplants are able to induce robust remyelination with normal compact myelin ultrastructure and organisation in dysmyelinated mice [122]. However, there are significant hurdles associated with cell transplantation [123]. MS is a multifocal chronic disease and would therefore probably require cell transplantation into all areas of demyelination throughout the course of the disease, as evidence from experimental animal models suggests that transplanted glial progenitor cells may have limited migration potential [124]. Controlling the proliferation and differentiation process of transplanted cells may also be difficult; the cells could migrate inappropriately or proliferate excessively (perhaps forming tumours), and fail to remyelinate. Even if we surmount these problems in our animal models, as they may not reflect the exact human pathology, this may lead to different cell behaviour and effect in clinical trials of transplants in humans. Patients would need immunosuppression if the source of transplanted OPCs is human embryonic stem cells; however, OPCs can now also be derived from patient-specific induced pluripotent stem (iPS) cells (albeit slowly), and these mature into oligodendrocytes, forming functional myelin around axons at least in mouse brain. This may provide an alternative to strong immune suppression [125]. However, these techniques are still being developed, and their safety and efficacy remain unknown.

\section{Discussion}

While only a very few remyelination-enhancing therapies are currently being tested in clinical trials, recent preclinical research has identified many potential targets that enhance OPC migration to the site of injury and differentiation into myelinating oligodendrocytes, both of which may improve remyelination in vivo. In addition, much work is focusing on the underlying pathology in MS, especially to elucidate why remyelination is inefficient in the human condition compared with other animals. The grouping of these targets into two classes - the promigration and the pro-differentiation pathways - provides both advantages and disadvantages. There is now a diversity of targets, but how do we determine which drug to use for which MS lesion in which patient and at what time? For example, anti-LINGO-1 antibodies and RXR agonists may enhance remyelination in lesions containing OPCs with impaired differentiation, but not in OPC-deficient lesions. Conversely, a Sema3A-neuropilin1 interaction inhibitor may help in treating lesions with a lack of progenitor cells only. As MS patients mostly present with a mixture of both types of lesions, should we just treat with molecules from both groups or could this be detrimental? Some molecules that promote migration, e.g. PDGF, also inhibit differentiation, which may be counterproductive at the wrong time. Understanding the time course of remyelination in human MS, which will be different from in experimental models, will be critical here, but this is where we find another hurdle: remyelination in humans cannot yet be reliably measured directly. Several studies have attempted to use MRI measures in order to quantify myelination [126-129], but the resolution of these imaging techniques is not yet good enough to distinguish between varying degrees of 
remyelination/demyelination and axonal damage. The outcome of brain atrophy measured by MRI is correlated with disease progression, but this is downstream of, and maybe also in part unrelated to, the remyelination effect and takes time to change. In the laboratory setting, using experimental models of demyelination, remyelination is quantified by counting the number of axons with a myelin sheath and measuring the thickness of this myelin using electron microscopy. This is the (time-consuming) gold standard for assessing the extent of remyelination; however, it is useless for in vivo quantification and human clinical trials. An alternative biomarker, preferably in the serum or perhaps the CSF, will be critical to help assess remyelination, but identifying a suitable candidate remains a challenge. CSF neurofilament levels may represent neurodegeneration in MS [130], but, although potentially useful, this is still downstream of remyelination, and not a direct measure of it. Furthermore, determining which MS lesions have sufficient or few OPCs in vivo is currently impossible.

In spite of this lack of a robust biomarker of remyelination, clinical trials studying potential pro-remyelinating therapies are in progress, using the outcomes of MR measures of brain atrophy, retinal nerve layer thickness (using optical coherence tomography) and clinical disability-all surrogate markers. However, pharma are prepared to embark on such costly and time-consuming trials due to the importance of trying to find a treatment for the unmet need of treatment to avoid progressive MS. Design of these clinical trials raises still more problems with regard to subject selection and timing of treatments: which MS patients with what disease course (RRMS vs. SPMS vs. PPMS) should be treated and when? When would we expect to see a difference? How can we deliver the drug? The answers to these questions are difficult and compromises will be made and lessons learnt from early trials. As we believe that endogenous remyelination is reasonably efficient early in RRMS, perhaps pro-remyelination therapy is only needed later in the relapsing-remitting stage or at the secondary progressive stage, when disability accumulates. However, we also know that neurodegeneration occurs early and perhaps, by this time, some lesions may be chronically demyelinated and not amenable to this therapy. When progressive disease is established, is this the time to initiate such therapy, or is this too little, too late? Or, by starting such treatment early, would we inflict drugs (which inevitably have side effects) on patients who would do better without our intervention? Is it also wise to combine immunomodulator and pro-remyelinating drugs? We know that some aspects of inflammation are required for successful remyelination, at least in part due to activation of microglia/macrophages to both clear the debris and produce pro-regenerative factors $[53,54,131]$. The only way to resolve these questions is to improve imaging of such lesions, perhaps by positron emission tomography (PET) imaging or new MR sequences. Techniques to trace OPCs after relapses or during progressive disease in MS patients may give insight into the time course of OPC recruitment and differentiation and inform us when remyelinationpromoting targets may have a positive effect.

In addition to choosing the correct patients, and treating at the most appropriate time, how long should each trial be? We do not know the tempo of remyelination in humans, but it is likely to be over months, and the subsequent effect on protection from neurodegeneration may only become obvious over years. Long clinical trials are very expensive but probably currently necessary using our current outcome measures of brain atrophy or accumulation of disability.

Designing a pro-remyelinating drug from the targets identified to be appropriate for patients is also challenging. Ideally, the drug would be oral, long-acting, selective for remyelination in the CNS and with no other systemic side effects. Currently licensed MS treatments act by modulating the immune system and are therefore not required to enter the CNS. However, compounds that promote remyelination need to pass through the bloodbrain barrier, which complicates their design-for example, the amount of antibody reaching the CNS when given systemically will be low. To get around this problem, we may consider direct delivery of potential drug candidates into the CNS, either via the CSF or even perhaps intra-nasally, and chemists are trying to design ways of packaging molecules so that they cross the blood-brain barrier more easily, thus trying to make the treatments more effective when given systemically and making them patient friendly.

All of these complex considerations are necessary in order to design the best clinical trials that help us to understand whether potential candidate drugs aid remyelination in human MS patients to provide putative treatments that slow disease.

\section{Conclusions}

Remyelination-enhancing therapies for patients with MS are not yet reality, but we believe they will reduce disease progression and disability. Preclinical research has identified multiple targets affecting remyelination in experimental animal models of demyelinating disease, and early stage human clinical trials have started. However, these studies need validation, careful planning due to the lack of easy and robust biomarkers for remyelination, and time, which is frustrating (but necessary) for patients and doctors alike. 
Acknowledgments Dr Williams and Dr Münzel have no conflicts of interest that are directly relevant to this review, and no funding was received for the paper. AW is currently funded by the Chief Scientist's Office, Scotland and EJM by the Centre for Clinical Brain Sciences, University of Edinburgh.

Open Access This article is distributed under the terms of the Creative Commons Attribution Noncommercial License which permits any noncommercial use, distribution, and reproduction in any medium, provided the original author(s) and the source are credited.

\section{References}

1. Pugliatti M, Sotgiu S, Rosati G. The worldwide prevalence of multiple sclerosis. Clin Neurol Neurosurg. 2002;104(3):182-91.

2. Costenbader KH, Gay S, Alarcon-Riquelme ME, et al. Genes, epigenetic regulation and environmental factors: which is the most relevant in developing autoimmune diseases? Autoimmun Rev. 2012;11(8):604-9.

3. Lucchinetti C, Bruck W, Parisi J, et al. A quantitative analysis of oligodendrocytes in multiple sclerosis lesions. A study of 113 cases. Brain. 1999;122(Pt 12):2279-95.

4. Noseworthy JH, Lucchinetti C, Rodriguez M, et al. Multiple sclerosis. N Engl J Med. 2000;343(13):938-52.

5. Chastain EM, Miller SD. Molecular mimicry as an inducing trigger for CNS autoimmune demyelinating disease. Immunol Rev. 2012;245(1):227-38.

6. Wu GF, Alvarez E. The immunopathophysiology of multiple sclerosis. Neurol Clin. 2011;29(2):257-78.

7. Compston A, Coles A. Multiple sclerosis. Lancet. 2008;372(9648):1502-17.

8. Funfschilling U, Supplie LM, Mahad D, et al. Glycolytic oligodendrocytes maintain myelin and long-term axonal integrity. Nature. 2012;485(7399):517-21.

9. Lee Y, Morrison BM, Li Y, et al. Oligodendroglia metabolically support axons and contribute to neurodegeneration. Nature. 2012;487(7408):443-8

10. Ferguson B, Matyszak MK, Esiri MM, et al. Axonal damage in acute multiple sclerosis lesions. Brain. 1997;120(Pt 3):393-9.

11. Trapp BD, Peterson J, Ransohoff RM, et al. Axonal transection in the lesions of multiple sclerosis. N Engl J Med. 1998; 338(5):278-85.

12. Dutta R, McDonough J, Yin X, et al. Mitochondrial dysfunction as a cause of axonal degeneration in multiple sclerosis patients. Ann Neurol. 2006;59(3):478-89.

13. Lovas G, Szilagyi N, Majtenyi K, et al. Axonal changes in chronic demyelinated cervical spinal cord plaques. Brain. 2000;123(Pt 2):308-17.

14. Bitsch A, Schuchardt J, Bunkowski S, et al. Acute axonal injury in multiple sclerosis. Correlation with demyelination and inflammation. Brain. 2000;123(Pt 6):1174-83.

15. Kuhlmann T, Lingfeld G, Bitsch A, et al. Acute axonal damage in multiple sclerosis is most extensive in early disease stages and decreases over time. Brain. 2002;125(Pt 10):2202-12.

16. Khademi M, Dring AM, Gilthorpe JD, et al. Intense inflammation and nerve damage in early multiple sclerosis subsides at older age: a reflection by cerebrospinal fluid biomarkers. PLoS One. 2013;8(5):e63172.

17. Vercellino M, Masera S, Lorenzatti M, et al. Demyelination, inflammation, and neurodegeneration in multiple sclerosis deep gray matter. J Neuropathol Exp Neurol. 2009;68(5):489-502.

18. Mistry N, Abdel-Fahim R, Mougin O, et al. Cortical lesion load correlates with diffuse injury of multiple sclerosis normal appearing white matter. Mult Scler. Epub 15 July 2013.
19. Crespy L, Zaaraoui W, Lemaire M, et al. Prevalence of grey matter pathology in early multiple sclerosis assessed by magnetization transfer ratio imaging. PLoS One. 2011;6(9):e24969.

20. Tallantyre EC, Bo L, Al-Rawashdeh O, et al. Greater loss of axons in primary progressive multiple sclerosis plaques compared to secondary progressive disease. Brain. 2009;132(Pt 5):1190-9.

21. Calabrese M, Agosta F, Rinaldi F, et al. Cortical lesions and atrophy associated with cognitive impairment in relapsingremitting multiple sclerosis. Arch Neurol. 2009;66(9):1144-50.

22. Tallantyre EC, Bo L, Al-Rawashdeh O, et al. Clinico-pathological evidence that axonal loss underlies disability in progressive multiple sclerosis. Mult Scler. 2010;16(4):406-11.

23. Trapp BD, Ransohoff R, Rudick R. Axonal pathology in multiple sclerosis: relationship to neurologic disability. Curr Opin Neurol. 1999;12(3):295-302.

24. Craner MJ, Lo AC, Black JA, et al. Abnormal sodium channel distribution in optic nerve axons in a model of inflammatory demyelination. Brain. 2003;126(Pt 7):1552-61.

25. England JD, Gamboni F, Levinson SR, et al. Changed distribution of sodium channels along demyelinated axons. Proc Natl Acad Sci USA. 1990;87(17):6777-80.

26. Nikic I, Merkler D, Sorbara C, et al. A reversible form of axon damage in experimental autoimmune encephalomyelitis and multiple sclerosis. Nat Med. 2011;17(4):495-9.

27. Smith KJ, Lassmann H. The role of nitric oxide in multiple sclerosis. Lancet Neurol. 2002;1(4):232-41.

28. Craner MJ, Newcombe J, Black JA, et al. Molecular changes in neurons in multiple sclerosis: altered axonal expression of Nav1.2 and Nav1.6 sodium channels and $\mathrm{Na}^{+} / \mathrm{Ca}^{2+}$ exchanger. Proc Natl Acad Sci USA. 2004;101(21):8168-73.

29. Howell OW, Palser A, Polito A, et al. Disruption of neurofascin localization reveals early changes preceding demyelination and remyelination in multiple sclerosis. Brain. 2006;129(Pt 12): 3173-85.

30. Coman I, Aigrot MS, Seilhean D, et al. Nodal, paranodal and juxtaparanodal axonal proteins during demyelination and remyelination in multiple sclerosis. Brain. 2006;129(Pt 12):3186-95.

31. Zambonin JL, Zhao C, Ohno N, et al. Increased mitochondrial content in remyelinated axons: implications for multiple sclerosis. Brain. 2011;134(Pt 7):1901-13.

32. Irvine KA, Blakemore WF. Remyelination protects axons from demyelination-associated axon degeneration. Brain. 2008; 131(Pt 6):1464-77.

33. Duncan ID, Brower A, Kondo Y, et al. Extensive remyelination of the CNS leads to functional recovery. Proc Natl Acad Sci USA. 2009;106(16):6832-6.

34. Liebetanz D, Merkler D. Effects of commissural de- and remyelination on motor skill behaviour in the cuprizone mouse model of multiple sclerosis. Exp Neurol. 2006;202(1):217-24.

35. Murray PD, McGavern DB, Sathornsumetee S, et al. Spontaneous remyelination following extensive demyelination is associated with improved neurological function in a viral model of multiple sclerosis. Brain. 2001;124(Pt 7):1403-16.

36. Patrikios P, Stadelmann C, Kutzelnigg A, et al. Remyelination is extensive in a subset of multiple sclerosis patients. Brain. 2006;129(Pt 12):3165-72.

37. Banati RB, Newcombe J, Gunn RN, et al. The peripheral benzodiazepine binding site in the brain in multiple sclerosis: quantitative in vivo imaging of microglia as a measure of disease activity. Brain. 2000;123(Pt 11):2321-37.

38. Manrique-Hoyos N, Jurgens T, Gronborg M, et al. Late motor decline after accomplished remyelination: impact for progressive multiple sclerosis. Ann Neurol. 2012;71(2):227-44.

39. Lindner M, Fokuhl J, Linsmeier F, et al. Chronic toxic demyelination in the central nervous system leads to axonal damage despite remyelination. Neurosci Lett. 2009;453(2):120-5. 
40. Richardson WD, Young KM, Tripathi RB, et al. NG2-glia as multipotent neural stem cells: fact or fantasy? Neuron. 2011;70(4):661-73.

41. Franklin RJ, Gilson JM, Blakemore WF. Local recruitment of remyelinating cells in the repair of demyelination in the central nervous system. J Neurosci Res. 1997;50(2):337-44.

42. Menn B, Garcia-Verdugo JM, Yaschine C, et al. Origin of oligodendrocytes in the subventricular zone of the adult brain. J Neurosci. 2006;26(30):7907-18.

43. Nait-Oumesmar B, Decker L, Lachapelle F, et al. Progenitor cells of the adult mouse subventricular zone proliferate, migrate and differentiate into oligodendrocytes after demyelination. Eur J Neurosci. 1999;11(12):4357-66.

44. Zawadzka M, Rivers LE, Fancy SP, et al. CNS-resident glial progenitor/stem cells produce Schwann cells as well as oligodendrocytes during repair of CNS demyelination. Cell Stem Cell. 2010;6(6):578-90.

45. Mirsky R, Woodhoo A, Parkinson DB, et al. Novel signals controlling embryonic Schwann cell development, myelination and dedifferentiation. J Peripher Nerv Syst. 2008;13(2):122-35.

46. Keirstead HS, Blakemore WF. Identification of post-mitotic oligodendrocytes incapable of remyelination within the demyelinated adult spinal cord. J Neuropathol Exp Neurol. 1997;56(11):1191-201.

47. Targett MP, Sussman J, Scolding N, et al. Failure to achieve remyelination of demyelinated rat axons following transplantation of glial cells obtained from the adult human brain. Neuropathol Appl Neurobiol. 1996;22(3):199-206.

48. Groves AK, Barnett SC, Franklin RJ, et al. Repair of demyelinated lesions by transplantation of purified $\mathrm{O}-2 \mathrm{~A}$ progenitor cells. Nature. 1993;362(6419):453-5.

49. Gensert JM, Goldman JE. Endogenous progenitors remyelinate demyelinated axons in the adult CNS. Neuron. 1997;19(1): 197-203.

50. Patani R, Balaratnam M, Vora A, et al. Remyelination can be extensive in multiple sclerosis despite a long disease course. Neuropathol Appl Neurobiol. 2007;33(3):277-87.

51. Goldschmidt T, Antel J, Konig FB, et al. Remyelination capacity of the MS brain decreases with disease chronicity. Neurology. 2009;72(22):1914-21.

52. Boyd A, Zhang H, and Williams A. Insufficient OPC migration into demyelinated lesions is a cause of poor remyelination in MS and mouse models. Acta Neuropathol. 2013;125(6):841-59.

53. Kotter MR, Li WW, Zhao C, et al. Myelin impairs CNS remyelination by inhibiting oligodendrocyte precursor cell differentiation. J Neurosci. 2006;26(1):328-32.

54. Miron VE, Boyd A, Zhao JW, et al. M2 microglia and macrophages drive oligodendrocyte differentiation during CNS remyelination. Nat Neurosci. 2013;16(9):1211-8.

55. Williams A, Piaton G, Lubetzki C. Astrocytes-friends or foes in multiple sclerosis? Glia. 2007;55(13):1300-12.

56. Gledhill RF, Harrison BM, McDonald WI. Pattern of remyelination in the CNS. Nature. 1973;244(5416):443-4.

57. Gledhill RF, McDonald WI. Morphological characteristics of central demyelination and remyelination: a single-fiber study. Ann Neurol. 1977;1(6):552-60.

58. Perier O, Gregoire A. Electron microscopic features of multiple sclerosis lesions. Brain. 1965;88(5):937-52.

59. Prineas JW, Connell F. Remyelination in multiple sclerosis. Ann Neurol. 1979;5(1):22-31.

60. Raine CS, Wu E. Multiple sclerosis: remyelination in acute lesions. J Neuropathol Exp Neurol. 1993;52(3):199-204.

61. Coelho RP, Payne SG, Bittman R, et al. The immunomodulator FTY720 has a direct cytoprotective effect in oligodendrocyte progenitors. J Pharmacol Exp Ther. 2007;323(2):626-35.
62. Miron VE, Jung CG, Kim HJ, et al. FTY720 modulates human oligodendrocyte progenitor process extension and survival. Ann Neurol. 2008;63(1):61-71.

63. Miron VE, Ludwin SK, Darlington PJ, et al. Fingolimod (FTY720) enhances remyelination following demyelination of organotypic cerebellar slices. Am J Pathol. 2010;176(6): 2682-94.

64. Hu Y, Lee X, Ji B, et al. Sphingosine 1-phosphate receptor modulator fingolimod (FTY720) does not promote remyelination in vivo. Mol Cell Neurosci. 2011;48(1):72-81.

65. Khatri B, Barkhof F, Comi G, et al. Comparison of fingolimod with interferon beta-1a in relapsing-remitting multiple sclerosis: a randomised extension of the TRANSFORMS study. Lancet Neurol. 2011;10(6):520-9.

66. Robak T. Emerging monoclonal antibodies and related agents for the treatment of chronic lymphocytic leukemia. Future Oncol. 2013;9(1):69-91.

67. Zinzani PL, Corradini P, Gallamini A, et al. Overview of alemtuzumab therapy for the treatment of T-cell lymphomas. Leuk Lymphoma. 2012;53(5):789-95.

68. Cohen JA, Coles AJ, Arnold DL, et al. Alemtuzumab versus interferon beta 1a as first-line treatment for patients with relapsing-remitting multiple sclerosis: a randomised controlled phase 3 trial. Lancet. 2012;380(9856):1819-28.

69. Coles AJ, Twyman CL, Arnold DL, et al. Alemtuzumab for patients with relapsing multiple sclerosis after disease-modifying therapy: a randomised controlled phase 3 trial. Lancet. 2012;380(9856):1829-39.

70. Jones JL, Anderson JM, Phuah CL, et al. Improvement in disability after alemtuzumab treatment of multiple sclerosis is associated with neuroprotective autoimmunity. Brain. 2010;133(Pt 8):2232-47.

71. Linker RA, Lee DH, Ryan S, et al. Fumaric acid esters exert neuroprotective effects in neuroinflammation via activation of the Nrf2 antioxidant pathway. Brain. 2011;134(Pt 3):678-92.

72. Fox RJ, Miller DH, Phillips JT, et al. Placebo-controlled phase 3 study of oral BG-12 or glatiramer in multiple sclerosis. N Engl J Med. 2012;367(12):1087-97.

73. Gold R, Kappos L, Arnold DL, et al. Placebo-controlled phase 3 study of oral BG-12 for relapsing multiple sclerosis. N Engl J Med. 2012;367(12):1098-107.

74. Zhornitsky S, Wee Yong V, Koch MW, et al. Quetiapine fumarate for the treatment of multiple sclerosis: focus on myelin repair. CNS Neurosci Ther. 2013;19(10):737-44.

75. Xiao L, Xu H, Zhang Y, et al. Quetiapine facilitates oligodendrocyte development and prevents mice from myelin breakdown and behavioral changes. Mol Psychiatry. 2008;13(7):697-708.

76. Bi X, Zhang Y, Yan B, et al. Quetiapine prevents oligodendrocyte and myelin loss and promotes maturation of oligodendrocyte progenitors in the hippocampus of global cerebral ischemia mice. J Neurochem. 2012;123(1):14-20.

77. Mei F, Guo S, He Y, et al. Quetiapine, an atypical antipsychotic, is protective against autoimmune-mediated demyelination by inhibiting effector $\mathrm{T}$ cell proliferation. PLoS One. 2012; 7(8):e42746.

78. Zhang Y, Zhang H, Wang L, et al. Quetiapine enhances oligodendrocyte regeneration and myelin repair after cuprizoneinduced demyelination. Schizophr Res. 2012;138(1):8-17.

79. Mi S, Lee X, Shao Z, et al. LINGO-1 is a component of the Nogo-66 receptor/p75 signaling complex. Nat Neurosci. 2004; 7(3):221-8.

80. Mi S, Hu B, Hahm K, et al. LINGO-1 antagonist promotes spinal cord remyelination and axonal integrity in MOG-induced experimental autoimmune encephalomyelitis. Nat Med. 2007;13(10):1228-33. 
81. Mi S, Miller RH, Tang W, et al. Promotion of central nervous system remyelination by induced differentiation of oligodendrocyte precursor cells. Ann Neurol. 2009;65(3):304-15.

82. Pepinsky RB, Walus L, Shao Z, et al. Production of a PEGylated Fab' of the anti-LINGO-1 Li33 antibody and assessment of its biochemical and functional properties in vitro and in a rat model of remyelination. Bioconjug Chem. 2011;22(2):200-10.

83. Cadavid D, Butzkueven H, Yaou L et al. Effect of LINGO-1 blockade on optic nerve axonal injury in MOG-EAE rodent models. Neurology. 2013;80(Meeting Abstracts 1): P05.186.

84. Mitsunaga Y, Ciric B, Van Keulen V, et al. Direct evidence that a human antibody derived from patient serum can promote myelin repair in a mouse model of chronic-progressive demyelinating disease. FASEB J. 2002;16(10):1325-7.

85. Pirko I, Ciric B, Gamez J, et al. A human antibody that promotes remyelination enters the CNS and decreases lesion load as detected by T2-weighted spinal cord MRI in a virus-induced murine model of MS. FASEB J. 2004;18(13):1577-9.

86. Ciric B, Van Keulen V, Paz Soldan M, et al. Antibody-mediated remyelination operates through mechanism independent of immunomodulation. J Neuroimmunol. 2004;146(1-2): 153-61.

87. Howe CL, Bieber AJ, Warrington AE, et al. Antiapoptotic signaling by a remyelination-promoting human antimyelin antibody. Neurobiol Dis. 2004;15(1):120-31.

88. Watzlawik J, Holicky E, Edberg DD, et al. Human remyelination promoting antibody inhibits apoptotic signaling and differentiation through Lyn kinase in primary rat oligodendrocytes. Glia. 2010;58(15):1782-93.

89. Watzlawik JO, Warrington AE, Rodriguez M. PDGF is required for remyelination-promoting IgM stimulation of oligodendrocyte progenitor cell proliferation. PLoS One. 2013;8(2):e55149.

90. Chang A, Tourtellotte WW, Rudick R, et al. Premyelinating oligodendrocytes in chronic lesions of multiple sclerosis. N Engl J Med. 2002;346(3):165-73.

91. Redwine JM, Armstrong RC. In vivo proliferation of oligodendrocyte progenitors expressing PDGFalphaR during early remyelination. J Neurobiol. 1998;37(3):413-28.

92. Woodruff RH, Fruttiger M, Richardson WD, et al. Plateletderived growth factor regulates oligodendrocyte progenitor numbers in adult CNS and their response following CNS demyelination. Mol Cell Neurosci. 2004;25(2):252-62.

93. Allamargot C, Pouplard-Barthelaix A, Fressinaud C. A single intracerebral microinjection of platelet-derived growth factor (PDGF) accelerates the rate of remyelination in vivo. Brain Res. 2001;918(1-2):28-39.

94. Spassky N, de Castro F, Le Bras B, et al. Directional guidance of oligodendroglial migration by class 3 semaphorins and netrin-1. J Neurosci. 2002;22(14):5992-6004.

95. Sugimoto Y, Taniguchi M, Yagi T, et al. Guidance of glial precursor cell migration by secreted cues in the developing optic nerve. Development. 2001;128(17):3321-30.

96. Williams A, Piaton G, Aigrot MS, et al. Semaphorin 3A and 3F: key players in myelin repair in multiple sclerosis? Brain. 2007;130(Pt 10):2554-65.

97. Piaton G, Aigrot MS, Williams A, et al. Class 3 semaphorins influence oligodendrocyte precursor recruitment and remyelination in adult central nervous system. Brain. 2011;134(Pt 4):1156-67.

98. Omari KM, John G, Lango R, et al. Role for CXCR2 and CXCL1 on glia in multiple sclerosis. Glia. 2006;53(1):24-31.

99. Omari KM, John GR, Sealfon SC, et al. CXC chemokine receptors on human oligodendrocytes: implications for multiple sclerosis. Brain. 2005;128(Pt 5):1003-15.

100. Omari KM, Lutz SE, Santambrogio L, et al. Neuroprotection and remyelination after autoimmune demyelination in mice that inducibly overexpress CXCL1. Am J Pathol. 2009;174(1):164-76.

101. John GR, Shankar SL, Shafit-Zagardo B, et al. Multiple sclerosis: re-expression of a developmental pathway that restricts oligodendrocyte maturation. Nat Med. 2002;8(10):1115-21.

102. Givogri MI, Costa RM, Schonmann V, et al. Central nervous system myelination in mice with deficient expression of Notch1 receptor. J Neurosci Res. 2002;67(3):309-20.

103. Wang S, Sdrulla AD, diSibio G, et al. Notch receptor activation inhibits oligodendrocyte differentiation. Neuron. 1998;21(1): 63-75.

104. Zhang Y, Argaw AT, Gurfein BT, et al. Notch1 signaling plays a role in regulating precursor differentiation during CNS remyelination. Proc Natl Acad Sci USA. 2009;106(45):19162-7.

105. Zhang Y, Zhang J, Navrazhina K, et al. TGFbeta1 induces Jagged1 expression in astrocytes via ALK5 and Smad3 and regulates the balance between oligodendrocyte progenitor proliferation and differentiation. Glia. 2010;58(8):964-74.

106. Fancy SP, Baranzini SE, Zhao C, et al. Dysregulation of the Wnt pathway inhibits timely myelination and remyelination in the mammalian CNS. Genes Dev. 2009;23(13):1571-85.

107. Ye F, Chen Y, Hoang T, et al. HDAC1 and HDAC2 regulate oligodendrocyte differentiation by disrupting the beta-cateninTCF interaction. Nat Neurosci. 2009;12(7):829-38.

108. Lai K, Kaspar BK, Gage FH, et al. Sonic hedgehog regulates adult neural progenitor proliferation in vitro and in vivo. Nat Neurosci. 2003;6(1):21-7.

109. Machold R, Hayashi S, Rutlin M, et al. Sonic hedgehog is required for progenitor cell maintenance in telencephalic stem cell niches. Neuron. 2003;39(6):937-50.

110. Loulier K, Ruat M, Traiffort E. Increase of proliferating oligodendroglial progenitors in the adult mouse brain upon Sonic hedgehog delivery in the lateral ventricle. J Neurochem. 2006;98(2):530-42.

111. Ferent J, Zimmer C, Durbec P, et al. Sonic Hedgehog signaling is a positive oligodendrocyte regulator during demyelination. J Neurosci. 2013;33(5):1759-72.

112. Huang JK, Jarjour AA, Nait Oumesmar B, et al. Retinoid X receptor gamma signaling accelerates CNS remyelination. Nat Neurosci. 2011;14(1):45-53.

113. Yuen TJ, Johnson KR, Miron VE, et al. Identification of endothelin 2 as an inflammatory factor that promotes central nervous system remyelination. Brain. 2013;136(Pt 4):1035-47.

114. Gadea A, Aguirre A, Haydar TF, et al. Endothelin-1 regulates oligodendrocyte development. J Neurosci. 2009;29(32): 10047-62.

115. Sim FJ, McClain CR, Schanz SJ, et al. CD140a identifies a population of highly myelinogenic, migration-competent and efficiently engrafting human oligodendrocyte progenitor cells. Nat Biotechnol. 2011;29(10):934-41.

116. Patel JR, McCandless EE, Dorsey D, et al. CXCR4 promotes differentiation of oligodendrocyte progenitors and remyelination. Proc Natl Acad Sci USA. 2010;107(24):11062-7.

117. Ara J, See J, Mamontov P, et al. Bone morphogenetic proteins 4, 6 , and 7 are up-regulated in mouse spinal cord during experimental autoimmune encephalomyelitis. J Neurosci Res. 2008; 86(1):125-35.

118. Sabo JK, Aumann TD, Merlo D, et al. Remyelination is altered by bone morphogenic protein signaling in demyelinated lesions. J Neurosci. 2011;31(12):4504-10.

119. Sloane JA, Batt C, Ma Y, et al. Hyaluronan blocks oligodendrocyte progenitor maturation and remyelination through TLR2. Proc Natl Acad Sci USA. 2010;107(25):11555-60.

120. Sun Y, Xu CC, Li J, et al. Transplantation of oligodendrocyte precursor cells improves locomotion deficits in rats with spinal cord irradiation injury. PLoS One. 2013;8(2):e57534. 
121. Wu B, Sun L, Li P, et al. Transplantation of oligodendrocyte precursor cells improves myelination and promotes functional recovery after spinal cord injury. Injury. 2012;43(6):794-801.

122. Uchida N, Chen K, Dohse M, et al. Human neural stem cells induce functional myelination in mice with severe dysmyelination. Sci Transl Med. 2012;4(155):155ra136.

123. Lindvall O, Kokaia Z. Stem cells in human neurodegenerative disorders-time for clinical translation? J Clin Invest. 2010;120(1):29-40.

124. Blakemore WF, Chari DM, Gilson JM, et al. Modelling large areas of demyelination in the rat reveals the potential and possible limitations of transplanted glial cells for remyelination in the CNS. Glia. 2002;38(2):155-68.

125. Wang S, Bates J, Li X, et al. Human iPSC-derived oligodendrocyte progenitor cells can myelinate and rescue a mouse model of congenital hypomyelination. Cell Stem Cell. 2013;12(2):252-64.

126. Barkhof F, Bruck W, De Groot CJ, et al. Remyelinated lesions in multiple sclerosis: magnetic resonance image appearance. Arch Neurol. 2003;60(8):1073-81.
127. Brown RA, Narayanan S, Arnold DL. Segmentation of magnetization transfer ratio lesions for longitudinal analysis of demyelination and remyelination in multiple sclerosis. Neuroimage. 2012;66C:103-9.

128. Fox RJ, Cronin T, Lin $\mathrm{J}$, et al. Measuring myelin repair and axonal loss with diffusion tensor imaging. Am J Neuroradiol. 2011;32(1):85-91.

129. Sinnecker T, Mittelstaedt P, Dorr J, et al. Multiple sclerosis lesions and irreversible brain tissue damage: a comparative ultrahigh-field strength magnetic resonance imaging study. Arch Neurol. 2012;69(6):739-45.

130. Petzold A, Eikelenboom MI, Keir G, et al. The new global multiple sclerosis severity score (MSSS) correlates with axonal but not glial biomarkers. Mult Scler. 2006;12(3):325-8.

131. Kotter MR, Setzu A, Sim FJ, et al. Macrophage depletion impairs oligodendrocyte remyelination following lysolecithininduced demyelination. Glia. 2001;35(3):204-12. 